\title{
First Report of a de novo 10q23.31q23.33 Microdeletion: Obesity, Intellectual Disability and Microcephaly
}

\author{
Ayberk Turkyilmaz $^{\mathrm{a}}$ Erdal Kurnaz $^{\mathrm{b}}$ Atilla Cayir ${ }^{\mathrm{c}}$ \\ ${ }^{a}$ Department of Medical Genetics, Karadeniz Technical University Faculty of Medicine, Trabzon, Turkey; \\ ${ }^{b}$ Department of Pediatric Endocrinology, Dr Sami Ulus Obstetrics and Gynecology, Children's Health and \\ Disease Training and Research Hospital, Ankara, Turkey; 'Department of Pediatric Endocrinology, \\ Erzurum City Hospital, Erzurum, Turkey
}

\section{Established Facts}

- Intellectual disability (ID) is characterized by limited or insufficient development of mental abilities, including intellectual functioning impairments, such as learning and understanding cause-effect relationships.

- Obesity is a multifactorial disease in which both genetic and environmental factors play a role.

- The role of heredity in obesity has been reported to be between 40 and 70\%.

- Array-based comparative genomic hybridization (array-CGH) can detect CNVs in the whole genome at a higher resolution than conventional cytogenetic methods. Array-CGH is currently recommended as the first-tier genetic test for ID cases worldwide.

\section{Novel Insights}

- The present study is the first report of a de novo 10q23.31q23.33 microdeletion with obesity, ID and microcephaly.

- GPR120, HHEX, IDE, PPP1R3C, and MARCH5 genes in the deletion region are thought to explain the obesity phenotype of the patient.

- The KIF11 gene located in the deletion region is thought to explain microcephaly and the ID phenotype of the patient.

\section{Keywords}

Microcephaly · Obesity · Intellectual disability · 10q23.31q23.33 microdeletion · Array comparative genomic hybridization

\begin{abstract}
Intellectual disability (ID) is characterized by limited or insufficient development of mental abilities, including intellectual functioning impairments, such as learning and understanding cause-effect relationships. Some cases have ID as the only finding and are called isolated cases. Conversely,
\end{abstract}


cases accompanied by facial dysmorphism, microcephaly, autism spectrum disorder, epilepsy, obesity, and congenital anomalies are called syndromic developmental delay (DD)/ ID. Isolated and syndromic DD/ID cases show extreme genetic heterogeneity. Genetic etiology can be detected in approximately $40 \%$ of the cases, whereas chromosomal abnormalities are observed in $25 \%$. Obesity is a multifactorial disease in which both genetic and environmental factors play important roles. The role of heredity in obesity has been reported to be between 40 and 70\%. Array-based comparative genomic hybridization (array-CGH) can detect CNVs in the whole genome at a higher resolution than conventional cytogenetic methods. Array-CGH is currently recommended as the first-tier genetic test for ID cases worldwide. In the present study, we aimed to evaluate clinical, radiological, and genetic analyses of a 12-year and 4-monthold girl with microcephaly, ID, and obesity. In the array-CGH analysis, a 3.1-Mb deletion, arr[GRGh37] 10q23.31g23.33 (92745793_95937944) $\times 1$ was detected, and this alteration was evaluated to be pathogenic. We consider that haploinsufficiency of the candidate genes (GPR120, KIF11, EXOC6, CYP26A1, CYP26C1, and LGI1) in the deletion region may explain microcephaly, ID, obesity, seizures, and ophthalmological findings in our patient. The investigation of 10q23.31q23.33 microdeletion in cases with syndromic obesity may contribute to molecular genetic diagnosis.

(c) 2021 S. Karger AG, Base

\section{Introduction}

Intellectual disability (ID) is characterized by limited or insufficient development of mental abilities, including intellectual functioning impairments, such as learning and understanding cause-effect relationships [Choo et al., 2019]. ID individuals are often diagnosed in the early school-age period. Some cases have ID as the only finding and are called isolated cases. Conversely, cases accompanied by facial dysmorphism, microcephaly, autism spectrum disorder, epilepsy, obesity, and congenital anomalies are called syndromic developmental delay (DD)/ID [Kaufman et al., 2010]. Recent studies have shown that biological signaling pathways causing DD/ID, autism spectrum disorder, and epilepsy phenotypes are common. Additionally, the relationship between signaling pathways involved in early brain development, synaptic plasticity, neuronal migration and the phenotype result has been reported [Lo-Castro and Curatolo, 2014]. Isolated and syndromic DD/ID cases show extreme genetic heterogeneity. Genetic etiology can be detected in ap-

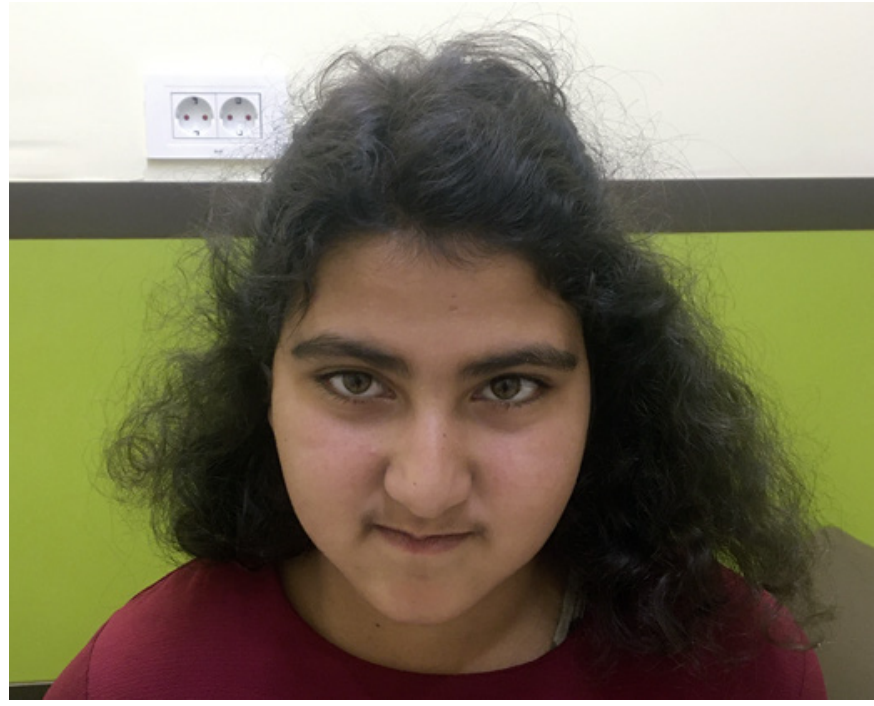

Fig. 1. Microcephaly, bitemporal narrowing, narrow forehead, upslanting palpebral fissures, thin upper lip, and small mouth were detected in the patient's dysmorphology examination.

proximately $40 \%$ of the cases, whereas chromosomal abnormalities are observed in 25\% [Moeschler et al., 2014; Miclea et al., 2015].

Obesity is a condition that affects $12 \%$ of the world population and causes life-threatening comorbidities such as cardiovascular disease, hypertension, type 2 diabetes mellitus, dyslipidemia, and cancer [GBD 2015 Obesity Collaborators et al., 2017] . Obesity is a multifactorial disease in which both genetic and environmental factors play important roles. The role of heredity in obesity has been reported to be between 40 and 70\% [Maes et al., 1997] . In terms of genetic etiology, obesity can be classified into 3 main groups: (1) monogenic obesity: the group associated with a defect of a gene in the leptin-melanocortin signaling pathway; (2) syndromic obesity: the group in which obesity is accompanied with neurodevelopmental anomalies and organ malformations, and (3) polygenic obesity: the group with the cumulative effect of multiple genes affecting energy intake and consumption. The majority of the patients with childhood obesity are found to have polygenic obesity [Thaker, 2017] .

Array-based comparative genomic hybridization (array-CGH) can detect CNVs in the whole genome at a higher resolution than conventional cytogenetic methods. CNVs are defined as changes $>1 \mathrm{~kb}$ resulting in an increase and/or decrease in genomic DNA [Lee et al., 2007]. In a review published by Miller et al. [2010], the diagnostic yield of array-CGH was $12.2 \%$ in $21,698 \mathrm{DD} /$ ID cases retrieved from 33 different studies. Array-CGH 
Fig. 2. Array-CGH result of chromosome 10. Enlargement on chromosome 10 shows loss of 10q23.31q23.33 corresponding to 3.1 $\mathrm{Mb}$ of DNA. $X$ axis corresponds to the genomic position in megabases of DNA and $Y$ axis shows the $\log _{2}$ ratio of signal intensity.

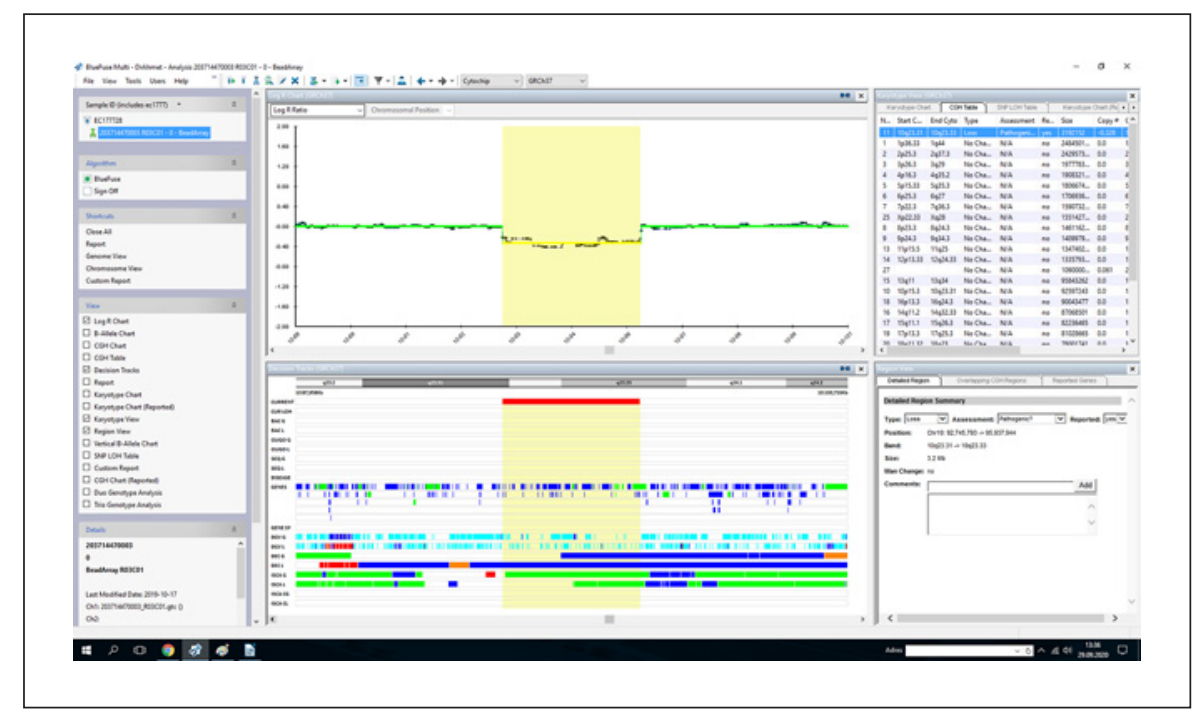

is currently recommended as the first-tier genetic test for ID cases worldwide [Miller et al., 2010].

In the present study, we aimed to evaluate clinical, radiological, and genetic analyses of a 12-year and 4-monthold girl with microcephaly, ID and obesity.

\section{Case Report}

Our patient was born via spontaneous vaginal delivery as the second child of healthy nonconsanguineous parents. At the age of 12 years and 4 months, physical examination revealed that the patient's height was $149 \mathrm{~cm}$ ( -0.91 SDS), weight was $60 \mathrm{~kg}$ (1.48 SDS), head circumference was $48 \mathrm{~cm}$ ( $-4.41 \mathrm{SDS})$, and body mass index was $27.03 \mathrm{~kg} / \mathrm{m}^{2}$ (2.09 SDS). Her puberty was assessed to be at Tanner stage 3. Physical examination showed microcephaly, bitemporal narrowing, a narrow forehead, upslanting palpebral fissures, thin upper lip, small mouth, and obesity (Fig. 1). The patient started walking when she was 1 year old and began to speak individual words at the age of 2 and built sentences at the age of 6 years. The patient had generalized tonic-clonic seizures 1-2 times a month for about 2 years from the age of 2 , and her seizures stopped without requirement for antiepileptic treatment. She has been receiving special education since she was 8 because of ID, but had no reading or writing skills. Our patient was followed up in the pediatric endocrinology clinic for about 3 years before genetic analysis due to her obesity. She was on a strict low-calorie diet, but she could not lose weight. Therefore, the fact that a genetic etiology may contribute to the obesity phenotype was considered.

Laboratory results demonstrated that plasma amino acids, plasma acylcarnitine profile, urine organic acid profile, lysosomal enzyme screening, plasma very-long-chain fatty acid level, lipid profile, $\mathrm{HbA1c}$, insulin, hemogram, and her vitamin B12 levels were all normal (Table 1). L-thyroxine therapy was initiated with a diagnosis of subclinical hypothyroidism (FT4: $0.92 \mathrm{ng} / \mathrm{dL}, \mathrm{TSH}: 13.7 \mathrm{mIU} /$ $\mathrm{mL}$ ). Thyroid function tests performed at 1 month after treatment (FT4: $0.92 \mathrm{ng} / \mathrm{dL}$, TSH: $2.42 \mathrm{mIU} / \mathrm{mL}$ ) were evaluated as normal.
Table 1. Laboratory results of the patient

\begin{tabular}{lc}
\hline & At presentation \\
\hline Fasting blood sugar, mg/dL & 74 \\
Insulin, mU/mL & 8.6 \\
HbA1C, \% & 5.4 \\
AST, U/L & 38 \\
ALT, U/L & 32 \\
Total cholesterol, mg/dL & 123 \\
HDL-cholesterol, mg/dL & 31.8 \\
LDL-cholesterol, mg/dL & 83 \\
Triglyceride, mg/dL & 121 \\
Free T4 ng/dL, normal range: $0.7-1.48$ & 0.92 \\
TSH mIu/mL, normal range: $0.35-4.94$ & 13.7 \\
antiTPO, U/mL (<5.6) & 0.13 \\
antiTG, U/mL $(<4.1)$ & 0.85 \\
\hline
\end{tabular}

Her echocardiograhy and EEG were normal. Abdominal ultrasound showed grade 1 hepatosteatosis. The patient had a normal hearing test, and her eye examination revealed antimetropia and astigmatism. Her brain MRI scan and orbital MRI were reported to be normal. Chromosome analysis of 20 metaphases at 450-band resolution performed by GTG-banding revealed a 46,XX normal female karyotype. Methylation analysis for Prader-Willi Syndrome was performed with SALSA MLPA Probemix ME028 Prader Willi/ Angelman kit (MRC-Holland, Amsterdam, The Netherlands) using the methylation-specific multiplex ligation-dependent probe amplification (MS-MLPA) method, and a normal methylation profile was detected. DNA isolation was performed using QIAamp ${ }^{\circledR}$ DNA Mini Kit (Qiagen GmbH, Hilden, Germany) from peripheral blood $(400 \mu \mathrm{L})$ for array-CGH analysis. Affymetrix CytoScan Optima $315 \mathrm{~K}$ arrays (Thermo Fisher Scientific, Waltham, MA, USA) were used according to the manufacturer's instructions to detect CNVs. Array-CGH results were evaluated using Chromosome Analysis Suite version 3.1.0 (Thermo Fisher Scientific). In the ar- 
ray-CGH analysis, a 3.1-Mb deletion, arr[GRGh37] 10q23.31g23.33 (92745793_95937944) $\times 1$, was detected, and this alteration was evaluated to be pathogenic (Fig. 2). Chromosome and array-CGH analyses of the healthy parents were normal, and the microdeletion in the proband was of de novo origin.

\section{Discussion}

A deletion of the chromosome $\operatorname{arr}[$ GRGh37] 10q23.31g23.33 (92745793_95937944) $\times 1$ region of approximately $3.1 \mathrm{Mb}$ has not been previously reported in the literature. In our patient, there are 19 OMIM genes in the 3.1-Mb deletion region; 8 of these genes, KIF11, CYP26C1, CEP55, GPR120, RBP4, PDE6C, LGI1, and PLCE1, have OMIM phenotypes. The heterozygous mutations in the KIF11 gene located in the chromosome 10q23.33 region are associated with the phenotype "microcephaly with or without chorioretinopathy, lymphedema, or mental retardation (OMIM \#152950)" in the OMIM database. Heterozygous mutations of the LGI1 gene located within the same region have been associated with the phenotype "epilepsy, familial temporal lobe, 1 (OMIM \#600512);" heterozygous mutations in the GPR120 gene with "obesity, susceptibility to (OMIM \#607514);" biallelic mutations in the CYP26C1 gene with "focal facial dermal dysplasia 4 (OMIM: \#614974);" homozygous mutations in the CEP55 gene with "multinucleated neurons, anhydramnios, renal dysplasia, cerebellar hypoplasia, and hydranencephaly (OMIM \#236500);" heterozygous mutations in the RBP4 gene with "microphthalmia, isolated, with coloboma 10 (OMIM \#616428), as well as its biallelic mutations with "retinal dystrophy, iris coloboma, and comedogenic acne syndrome (OMIM \#615147)," biallelic mutations in the PDE6C gene with "cone dystrophy 4 (OMIM \#613093)," and biallelic mutations in the PLCE1 gene with "nephrotic syndrome, type 3 (OMIM \#610725)" phenotypes. There are studies showing the relationship of HHEX and IDE genes with type 2 diabetes, obesity, and plasma lipid levels, although they have not been associated with any phenotypes in the OMIM database [Qian et al., 2012; Liu et al., 2014; Saini et al., 2018]. Moreover, the relationship of the PPP1R3C and MARCH5geneslocated in the chromosome 10q23.31q23.33 region with glucose intolerance, hyperinsulinemia, insulin resistance, and obesity were demonstrated with trials conducted in mice [Crosson et al., 2003; Bond et al., 2019].

In the literature, Malvezzi et al. [2018] reported that a father and his 2 children with microcephaly, mild ID, and chorioretinopathy findings had a 209-kb deletion, which included the KIF11 gene. The results of chorioretinopathy,

De novo 10q23.31q23.33 Microdeletion strabismus, hyperopia, and astigmatism were reported in the eye examinations of the patients performed after the deletion was detected [Malvezzi et al., 2018]. Meire et al. [2018] reported a deletion that included EXOC6, CYP26A1, and CYP26C1 with findings of nonsyndromic optic nerve aplasia in a father and his dizygotic twin children [Meire et al., 2011]. Zebrafish and mouse studies have shown that the exocist protein complex, including EXOC6, has a critical role in photoreceptor ciliogenesis and retinal development [Lobo et al., 2017]. In CYP26A1 and CY$\mathrm{P} 26 \mathrm{C} 1$ knockout mice, it was observed that the head circumference and the development of the eye and frontonasal region were significantly reduced [Uehara et al., 2007]. Following genetic analysis, antimetropia and astigmatism were detected in the detailed ophthalmological evaluation of our patient. Moreover, orbital MRI examination performed for detailed evaluation of the optic nerve was reported as normal. In our patient, the clinical picture may not have occurred due to incomplete penetrance, or it may occur at an older age. The patient was scheduled for followup for retinal anomalies that may develop in the future. It was considered that the KIF11, EXOC6, CYP26A1, and CY$P 26 C 1$ deletion could be responsible for developing microcephaly, ID, and ophthalmological results in our patient.

Our patient had generalized tonic-clonic seizures that started at the age of 2 and lasted for about 2 years. The microdeletion in the LGI1 gene located in the deletion region is known to be associated with autosomal dominant lateral temporal lobe epilepsy [Fanciulli et al., 2012]. Functional studies have shown that haploinsufficiency of the LGI1 gene causes an epilepsy phenotype. LGI1 links ADAM22 and ADAM23 proteins, which are epilepsy-related receptors in the brain. This gene also regulates the transynaptic protein complex composed of presynaptic potassium channels and postsynaptic AMPA receptors [Chabrol et al., 2010; Fukata et al., 2010; Yu et al., 2010]. The haploinsufficiency of the LGI1 gene may be contributing to the seizures of our patient.

The GPR120 gene, which is located in the deletion region, acts as a lipid sensor and provides energy balance. Gpr120-deficient mice have been demonstrated to develop obesity, glucose intolerance, and fatty liver when fed with a diet of high lipid content. Some heterozygous variants in the GPR120 gene have been reported to be associated with obesity in humans [Ichimura et al., 2012]. Furthermore, HHEX, IDE, PPP1R3C, and MARCH5 in the deletion region are associated with insulin and lipid metabolism and may indirectly affect body mass index [Crosson et al., 2003; Qian et al., 2012; Liu et al., 2014; Saini et al., 2018; Bond et al., 2019]. Deletion of these genes may be contributing to 
our patient's clinical presentation with obesity and hepatosteatosis.

We think that haploinsufficiency of the candidate genes (GPR120, KIF11, EXOC6, CYP26A1, CYP26C1, and LGI1) in the deletion region can explain microcephaly, ID, obesity, seizure, and ophthalmological findings in our patient. The investigation of 10q23.31q23.33 microdeletion in cases with syndromic obesity may contribute to the molecular genetic diagnosis.

\section{Statement of Ethics}

All experimental procedures were conducted in accordance with the principles of the Declaration of Helsinki, and informed written consent was obtained from patients or their guardians.

\section{Conflict of Interest Statement}

The authors have no conflicts of interest to declare.

\section{Funding Sources}

There was no funding relevant to this study.

\section{Author Contributions}

A.T. performed the array-CGH analysis as well as the segregation study and drafted the manuscript. E.K. and A.C. conducted the patient's physical examination and critically reviewed the manuscript.

\section{References}

Bond ST, Moody SC, Liu Y, Civelek M, Villanueva CJ, Gregorevic P, et al. The E3 ligase MARCH5 is a PPAR $\gamma$ target gene that regulates mitochondria and metabolism in adipocytes. Am J Physiol Endocrinol Metab. 2019; 316(2):E293-E304.

Chabrol E, Navarro V, Provenzano G, Cohen I, Dinocourt C, Rivaud-Péchoux S, et al. Electroclinical characterization of epileptic seizures in leucine-rich, glioma-inactivated 1-deficient mice. Brain. 2010;133(9):2749-62.

Choo YY, Agarwal P, How CH, Yeleswarapu SP. Developmental delay: identification and management at primary care level. Singapore Med J. 2019;60(3):119-23.

Crosson SM, Khan A, Printen J, Pessin JE, Saltiel AR. PTG gene deletion causes impaired glycogen synthesis and developmental insulin resistance. J Clin Invest. 2003;111(9):142332.

Fanciulli M, Santulli L, Errichiello L, Barozzi C, Tomasi L, Rigon L, et al. LGI1 microdeletion in autosomal dominant lateral temporal epilepsy. Neurology. 2012;78(17):1299-303.

Fukata Y, Lovero KL, Iwanaga T, Watanabe A, Yokoi N, Tabuchi K, et al. Disruption of LGI1-linked synaptic complex causes abnormal synaptic transmission and epilepsy. Proc Natl Acad Sci USA. 2010;107(8):3799-804.

GBD 2015 Obesity Collaborators, Afshin A, Forouzanfar MH, Reitsma MB, Sur P, Estep K, et al. Health effects of overweight and obesity in 195 countries over 25 years. N Engl J Med. 2017;377(1):13-27.

Ichimura A, Hirasawa A, Poulain-Godefroy O, Bonnefond A, Hara T, Yengo L, et al. Dysfunction of lipid sensor GPR120 leads to obesity in both mouse and human. Nature. 2012; 483(7389):350-4.
Kaufman L, Ayub M, Vincent JB. The genetic basis of non-syndromic intellectual disability: a review. J Neurodev Disord. 2010;2(4):182209.

Lee C, Iafrate AJ, Brothman AR. Copy number variations and clinical cytogenetic diagnosis of constitutional disorders. Nat Genet. 2007; 39(7 Suppl):48-54.

Liu S, Qian Y, Lu F, Dong M, Lin Y, Li H, et al. Genetic variants at $10 \mathrm{q} 23.33$ are associated with plasma lipid levels in a Chinese population. J Biomed Res. 2014;28(1):53-8.

Lobo GP, Fulmer D, Guo L, Zuo X, Dang Y, Kim $\mathrm{SH}$, et al. The exocyst is required for photoreceptor ciliogenesis and retinal development. J Biol Chem. 2017;292(36):14814-26.

Lo-Castro A, Curatolo P. Epilepsy associated with autism and attention deficit hyperactivity disorder: is there a genetic link?. Brain Dev. 2014; 36(3):185-93.

Maes HH, Neale MC, Eaves LJ. Genetic and environmental factors in relative body weight and human adiposity. Behav Genet. 1997;27(4): 325-51.

Malvezzi JV, H Magalhaes I, S Costa S, Otto PA, Rosenberg C, Bertola DR, et al. KIF11 microdeletion is associated with microcephaly, chorioretinopathy and intellectual disability. Hum Genome Var. 2018;5:18010.

Meire F, Delpierre I, Brachet C, Roulez F, Van Nechel C, Depasse F, et al. Nonsyndromic bilateral and unilateral optic nerve aplasia: first familial occurrence and potential implication of CYP26A1 and CYP26C1 genes. Mol Vis. 2011;17:2072-9
Miclea D, Peca L, Cuzmici Z, Pop IV. Genetic testing in patients with global developmental delay / intellectual disabilities. A review. Clujul Med. 2015;88(3):288-92.

Miller DT, Adam MP, Aradhya S, Biesecker LG, Brothman AR, Carter NP, et al. Consensus statement: chromosomal microarray is a firsttier clinical diagnostic test for individuals with developmental disabilities or congenital anomalies. Am J Hum Genet. 2010;86(5): 749-764.

Moeschler JB, Shevell M, Committee on Genetics. Comprehensive evaluation of the child with intellectual disability or global developmental delays. Pediatrics. 2014;134(3):e903-18.

Qian Y, Lu F, Dong M, Lin Y, Li H, Chen J, et al. Genetic variants of IDE-KIF11-HHEX at 10q23.33 associated with type 2 diabetes risk: a fine-mapping study in Chinese population. PLoS One. 2012;7(4):e35060.

Saini S, Walia GK, Sachdeva MP, Gupta V. Genetics of obesity and its measures in India. J Genet. 2018;97(4):1047-71.

Thaker VV. Genetic and Epigenetic causes of obesity. Adolesc Med State Art Rev. 2017;28(2): 379-405.

Uehara M, Yashiro K, Mamiya S, Nishino J, Chambon P, Dolle P, et al. CYP26A1 and CY$\mathrm{P} 26 \mathrm{C} 1$ cooperatively regulate anterior-posterior patterning of the developing brain and the production of migratory cranial neural crest cells in the mouse. Dev Biol. 2007; 302(2):399-411.

Yu YE, Wen L, Silva J, Li Z, Head K, Sossey-Alaoui $\mathrm{K}$, et al. Lgil null mutant mice exhibit myoclonic seizures and CA1 neuronal hyperexcitability. Hum Mol Genet. 2010;19(9):1702-11. 